\title{
Critical analysis of the classification of glaucomas issued by the European Glaucoma Society in 2008
}

This article was published in the following Dove Press journal:

Clinical Ophthalmology

23 January 2014

Number of times this article has been viewed

\section{Constantin-Dan Bordeianu}

Emergency Hospital Ploiesti, Prahova, Romania
Correspondence: Constantin-Dan Bordeianu

15, Cameliei St, BI 26, Sc B, Et I, Ap 26, Ploiesti, 10009I, Prahova, Romania Email bordmail3@yahoo.com and oftamedica.ro@gmail.com
Purpose: To critically analyze the 2008 European Glaucoma Society classification of glaucomas, in order to reveal its advantages and shortcomings.

Methods: The paper tries to determine the extent to which this classification is clear (being based on a coherent and consistently followed set of criteria), is comprehensive (framing all forms of glaucoma), helps to understand the sickness (using a logical framing system), and facilitates therapeutic decision making (offering direct therapeutic suggestions).

Results and conclusion: The paper shows that, compared with all the previous classifications, the 2008 European Glaucoma Society classification is one step ahead (in the way of classifying the group of secondary angle-closure glaucomas), two steps behind (in rejecting two useful categories of congenital glaucoma), and similar in several respects: that it is based on criticizable fundamental and secondary criteria that cannot cover all forms of sickness gathered at a particular crossing; that it uses several equally weighted criteria for one single crossing (division); that it frames one clinical entity in several clinical categories; that it does not reflect reality in some aspects; and that it does not offer direct therapeutic suggestions: after framing a case in a scheme built on the basis of gonioscopic observation, it requires a second stage of pathogenic analysis, so that the ophthalmologist is able to decide the correct treatment only in the third stage. All these considerations justify the efforts to find a new classification that will be able to correct the abovementioned shortcomings.

Keywords: glaucoma, classification of glaucomas, shortcomings of the 2008 EGS classification

\section{Introduction}

The role of any classification is to highlight the essential and defining element in a group of related phenomena, in order to facilitate some practical decision making. The major difficulty encountered in medical science is to find a criterion that can frame all forms of sickness in a coherent system, offering direct therapeutic suggestions.

Several attempts have been made to elaborate glaucoma $(G)$ classification, but only two have successfully survived (Table 1). Both these classifications reflected the contemporary level of knowledge. Donders ${ }^{1}$ could use only the little information offered by clinical practice, in a period when there were few means of investigation specific for G. The advent of gonioscopy threw light on a previously obscure domain, so that the gonioscopic classification ${ }^{2}$ was the first step toward understanding the pathogenic mechanism, explaining why some forms were silent and others so noisy. There were no essential differences between the two classifications. The names of some forms changed, but the content remained almost the same: "chronic simple G" became “open angle G” (OAG), while "congestive G” became "angle-closure G" 
Table I Donders' and gonioscopic classifications of glaucoma

\begin{tabular}{ll}
\hline Donders' clinical classification' & Gonioscopic classification $^{2}$ \\
\hline Congenital & Primary \\
Primary & Chronic simple \\
Chronic simple & Angle closure \\
Congestive & Secondary \\
Secondary & Open angle \\
& Angle closure \\
& Congenital \\
\hline
\end{tabular}

(ACG). Only one form, chronic ACG, changed its category: although it seemed to be chronic simple $\mathrm{G}$, it had a congestive pathogenic mechanism.

The gonioscopic classification was easily accepted because of this similitude. Without making considerable mental effort, its users got a much clearer explanation of the entities observed clinically. The new classification made them more familiar with the pathogeny of $\mathrm{G}$, making it easier to choose from the therapeutic options existing in the fifth decennium of the last century. In addition, it helped in the spread of gonioscopy, and as a consequence, the whole goniolens-producing industry supported the propagation of this new classification. In its form shown in Table 1, the gonioscopic classification remained almost unchanged for 50 years, although the volume of knowledge continuously increased, and the following new information emerged: G was no longer considered a sickness but a syndrome with too many forms to be easily accommodated within the narrow frame of the gonioscopic classification; the pathogeny of almost every form was clarified; and new therapeutic means had been described (both medical and surgical), so that almost every pathogenic form had its specific treatment. As a consequence, the need for a new classification that could integrate all this information became increasingly evident, and the first attempts appeared in the seventh decennium (Table 2).

From these, the classification suggested by Ourgaud and Etienne $^{3}$ was too simple for a sickness with so many forms, which is why it did not survive. The other classifications ${ }^{4-10}$ were variants of the gonioscopic classification, imparting clarity to some domains but retaining the confusion in other aspects. Therefore, none was able to replace the gonioscopic classification, whose main merit was its simplicity, together with an acceptable degree of clinical cover. Nevertheless, the discrepancy between the ever more complex reality and what the gonioscopic classification could cover grew with time; consequently, in the last years, the European Glaucoma Society (EGS) made several attempts to improve upon it, and the 2008 EGS classification (2008 EGSc) ${ }^{11}$ was the third and the last one. The purpose of this paper was to analyze the merits and drawbacks of the 2008 EGSc, a preparatory step before presenting a new classification of glaucomas, a pathogenic classification that avoids most of the drawbacks of the 2008 EGSc and maintains all its merits.

\section{Materials and methods}

The material is the 2008 EGSc, as described in Terminology and Guidelines for Glaucoma ${ }^{11}$ (Table 3). The method involved a critical analysis of the 2008 EGSc to determine the extent to which it is clear (being based on a coherent and consistently followed set of criteria), is comprehensive (framing all forms of glaucoma), helps to understand the sickness (using a logical framing system), and facilitates therapeutic decision making (offering direct therapeutic suggestions).

\section{Results - discussion}

After a careful study, my conclusion is that the 2008 EGSc is one step ahead, two steps behind, but similar in most respects - compared with all the previous classifications.

\section{The 2008 EGSc as one step ahead of the previous classifications}

This step is represented by the fact that the EGS finally tried to clarify the group of ACG, which contained too many forms characterized by angle closure (AC) despite the fact that the forms had different pathogenic mechanisms and sometimes

Table 2 The evolution of glaucoma classification in the last 50 years

\begin{tabular}{|c|c|c|c|c|c|c|c|}
\hline $\begin{array}{l}\text { Ourgaud and } \\
\text { Etienne }^{3}\end{array}$ & Krasnov $^{4}$ & $\begin{array}{l}\text { Saraux } \\
\text { and Biais }\end{array}$ & Etienne $^{6}$ & $\begin{array}{l}\text { Duke- } \\
\text { Elder }\end{array}$ & Bonamour $^{8}$ & $\begin{array}{l}\text { EGS } \\
1985-1998^{9}\end{array}$ & EGS 2004 10 \\
\hline Hypersecretion & Block in the angle & $\mathrm{C}$ & $\begin{array}{l}\mathrm{C} \text { (-OA, } \\
\text { - goniodys } \\
\text {-tardive) }\end{array}$ & C & $C(\mathrm{OA}, \mathrm{AC})$ & $\mathrm{C}(\mathrm{OA}, \mathrm{AC})$ & $\begin{array}{l}\text { C (-C, -infantile, } \\
\text {-associated) }\end{array}$ \\
\hline EVP increase & Block in the trabeculum & $P(O A, A C)$ & $\mathrm{OA}(\mathrm{P}, \mathrm{S})$ & $P(O A, A C)$ & $P(O A, A C)$ & $A C(P, S)$ & $\mathrm{OA}(\mathrm{P}, \mathrm{S})$ \\
\hline \multirow[t]{2}{*}{$\begin{array}{l}\text { Resistance } \\
\text { increase }\end{array}$} & $\begin{array}{l}\text { Block in the postschlemmal } \\
\text { vascular meshwork }\end{array}$ & S & $\mathrm{AC}(\mathrm{P}, \mathrm{S})$ & $S(O A, A C)$ & $\mathrm{S}(\mathrm{OA}, \mathrm{AC})$ & $\mathrm{OA}(\mathrm{P}, \mathrm{S})$ & $\mathrm{AC}(\mathrm{P}, \mathrm{S})$ \\
\hline & Hypersecretion & Mixed & & & & Mixed & \\
\hline
\end{tabular}

Abbreviations: AC, angle closure; C, congenital; EGS, European Glaucoma Society; EVP, episcleral venous pressure; goniodys, goniodysgenesis; OA, open angle; P, primary; S, secondary. 
Table 3 The 2008 European Glaucoma Society classification

I. Primary congenital $\mathrm{G}$

a. Primary congenital $\mathrm{G} /$ childhood $\mathrm{G}$

b. $G$ associated with congenital anomalies

2. $O A G$

a. Primary OAG (POAG)

i. Primary juvenile $G$

ii. Hypertensive adult $\mathrm{G}$

iii. Normotensive adult $\mathrm{G}$

iv. POAG suspect

v. Ocular hypertension

b. Secondary OAG

i. Caused by ocular disease (exfoliative, pigmentary, phacolytic, hemorrhagic, traumatic, uveitic, caused by tumors, associated with retinal detachment)

ii. latrogenic (after long-term corticosteroid therapy, after ocular surgery and laser)

iii. Caused by extrabulbar conditions (due to episcleral venous pressure increase)

3. ACG

a. Primary ACG (PACG)

i. Acute

ii. Intermittent

iii. Chronic

iv. Status after acute $A C$ attack

v. PAC suspect - AC risk

b. Secondary ACG

i. With pupillary block

ii. With pulling mechanism

iii. With pushing mechanism

Note: Data from European Glaucoma Society."

Abbreviations: AC, angle closure; ACG, angle closure glaucoma; G, glaucoma; OAG, open angle glaucoma; PACG, primary angle closure glaucoma; POAG, primary open angle glaucoma.

even opposite treatments. This created serious confusion, leading to therapeutic errors, since many times, the treatment for the most frequent form of ACG was automatically applied to any $\mathrm{G}$ case showing $\mathrm{AC}$.

The errors occurred because in all of the previous classifications, the framing of a new case in a scheme defined by gonioscopic aspect or by clinical behavior did not have direct therapeutic consequences for every case. After the case was framed in the scheme, the ophthalmologist had to consider pathogenic analysis in order to understand the mechanism of organic and functional alterations: in this analysis, he had to use all information, not only that offered by gonioscopy or by clinical behavior. Only then, in the third stage could the doctor choose the correct treatment for that particular case, after understanding its pathogenic mechanism. It is important to stress that the extent of correctness of the treatment depends on the extent of correctness of the pathogenic analysis. Unfortunately, doctors who avoid a detailed diagnostic procedure, who choose the shortest and easiest way have always existed; they refuse to make additional intellectual effort and skip the pathogenic analysis. Glad to have recognized the diagnostic category of even a subtype (eg, ACG in attack), some doctors could apply the treatment for the most frequent form of ACG in attack to any new case, regardless of its mechanism - simply because the angle is closed.

The fact that the 2008 EGSc tried to group the ACG forms according to the pathogenic mechanisms represents an important step forward, even if this action is limited to the secondary forms. Nevertheless, when we analyze the manner in which the secondary ACG forms are classified, we find that the manner is almost completely similar to my own pathogenic classification, first presented 27 years ago [Bordeianu CD. Clasificarea patogenică a glaucoamelor, Society of Medical Sciences (SSM) Bucureşti, 1986]. Later, the paper was included in the program of the 3rd EGS Symposium, Estoril, 1988 (unfortunately, Ceausescu's Securitate blocked my passport, so I could not present my paper); published in Romania in 1992 (in English); ${ }^{12}$ presented several times in Romania and as a free paper during the 12th European Society of Ophthalmology Congress in Stockholm, 1999. ${ }^{13}$ During these years, my pathogenic classification did not stir any interest, and to some extent, this was understandable because the classification was considerably ahead of the perception in those years. The 2008 EGSc reflected a change in perception, with the international ophthalmic community becoming aware of the gonioscopic criterion limitations and making the first move towards the use of the pathogenic criterion. In this scenario, I hope that the ophthalmic community will analyze, with a more positive mindset, the suggested classification, to be detailed in my next paper.

\section{The 2008 EGSc as two steps behind the previous classifications}

This is represented by the fact that, in congenital G, the 2008 EGSc has rejected two useful categories accepted by other contemporary classifications, ${ }^{9,10,14,15}$ without substituting with any other category/ies.

\section{The "open angle-angle closure" dichotomy has disappeared}

The "open angle (OA)-angle closure (AC)" dichotomy has disappeared in the 2008 EGSc, although many of the forms included by the 2008 EGSc in the congenital G group (Marfan syndrome, Weill-Marchesani syndrome, microcornea, aniridia, nanophthalmia, Sturge-Weber syndrome, Lowe syndrome, neurofibromatosis, Pierre Robin syndrome, 
homocystinuria, goniodysgenesis, broad thumb syndrome, and persistent hyperplastic primary vitreous) are also mentioned in the group of acquired ACG.

Logically, in a classification intended to clarify the way of thinking - especially of young ophthalmologists - any criterion used at a certain crossing should be valid for all the categories described at that particular crossing [I perceive the classification as a map with several successive "crossings," based on which, guided by every piece of available information, the doctor is able to find the right place for every new case, in such a way that the therapeutic decision is eased (the alternative term is "division")]. As consequence if the gonioscopic criterion is used in the group of acquired $\mathrm{G}$, it should be valid for the congenital G, too. Or, the 2008 EGSc does not use the gonioscopic criterion in congenital $\mathrm{G}$, in spite of the fact that the surgical techniques must target all pathogenic links, and that the angle narrowness may cause additional difficulties, imposing supplementary therapeutic gestures. Moreover, even if gonioscopy is not the only guiding technique for therapy and even if its findings must be corroborated with the information gathered by other investigation means, the gonioscopic indicator cannot be excluded without affecting the clarity and finality of a classification.

\section{The secondary forms have disappeared} for congenital G

The secondary forms have disappeared for congenital $G$, although the first line of the chapter on $\mathrm{G}$ classification in the Terminology and Guidelines for Glaucoma ${ }^{11}$ states that "all forms of $\mathrm{G}$ must be classified into primary and secondary forms." Nevertheless, after only seven lines, the existence of the secondary congenital $\mathrm{G}$ is denied, in spite of the fact that many forms of primary congenital $\mathrm{G}$ are later mentioned in the group of secondary acquired ACG. Moreover, the "secondary-to-rubella" G was included among the primary congenital "Gs associated with congenital anomalies," although the child's eye could have been perfectly normal without maternal rubella. Confused with other genetic anomalies, a young ophthalmologist could indicate avoidance of pregnancy to any couple who has produced a child with "primary congenital $\mathrm{G}$ associated with congenital anomalies" - the form where the 2008 EGSc places this manifestation. As the role of any classification is to prevent erroneous decisions by simplifying the way of thinking, these examples pose the question: Was denying the existence of secondary congenital $\mathrm{G}$ the right decision?

\section{The 2008 EGSc - similar in most respects with other previous classifications}

It maintains all the drawbacks of any previous classification:

\section{The 2008 EGSc uses criticizable fundamental criteria}

The 2008 EGSc uses three fundamental criteria for the first crossing (genetic, etiologic, and gonioscopic), all of which are subject to criticism.

The genetic criterion (distinguishing congenital from acquired G) might be criticized firstly because the trabecular meshwork alteration and the iridocorneal angle configuration - the characteristics that define the forms of acquired $\mathrm{G}$ - might be considered congenital anomalies with very late pathological manifestation.

Secondly, this criterion might explain many difficulties encountered in classifying congenital $\mathrm{G}$ forms. Most authors consider that the major characteristic of what is nowadays termed congenital $\mathrm{G}$ is the association of angle anomalies with ocular hypertension, manifested at an early age, on a distensible sclera, which explains the increase in ocular dimensions, Descemet membrane rupture, and fundus peculiarities. The isolated presence of angle anomalies at birth with no ocular hypertonia or its effects does not indicate congenital $\mathrm{G}$, in the same way that the late appearance of ocular hypertonia on a rigid sclera unable to expand does not indicate congenital $\mathrm{G}$, even if angle anomalies exist. On the other hand, buphthalmia always suggests congenital $\mathrm{G}$, even if angle anomalies cannot be observed because of some congenital or acquired problems of corneal transparency. If buphthalmia, the defining element in congenital G, may sometimes appear in acquired forms as well, while the concept of congenital $\mathrm{G}$ is opposite to that of acquired $\mathrm{G}$, it is possible to obtain more clarity if we reconsider the role of the genetic criterion in $\mathrm{G}$ classification. The exact manner will be suggested in my next paper.

The etiologic criterion (distinguishing primary from secondary $\mathrm{G}$ ) is subject to criticism because every form of $\mathrm{G}$ has a cause, which, for the moment, might be obscure. Furthermore, contemporary science has proved that there is a cause for almost any form of $\mathrm{G}$, even for the genetic anomalies.

The gonioscopic criterion (distinguishing OAG from $\mathrm{ACG}$ ) is also subject to criticism for four reasons. Firstly, the angle aspect is only a clinical sign, and its use as a fundamental criterion will produce a clinical classification, with all its drawbacks. It is not surprising that the gonioscopic classification greatly resembles Donders' one, since both are clinical classifications. 
Secondly, the gonioscopic criterion cannot cover all cases, and the most important manifestations are:

- tissue resistance alteration [I suggested this new term in order to cover the reality that the visual structures are characterized by an individual level of tissue resistance in front of aggressions, a level which may be normal, or altered]; this tissue resistance alteration may occur not only in OAG, but also, in ACG, not only in primary, but also, in secondary $\mathrm{G}$, and not only in normotensive, but also, in hypertensive cases, modulating the effects of pressure aggression;

- exogenous $\mathrm{G}$, which may develop in eyes with any degree of iridocorneal angle aperture and not only in an eye with $\mathrm{OA}$; and

- incipient stage of neovascular $\mathrm{G}$, in which the high pressure is not caused by AC with annular goniosynechia and pulling mechanism but by neovascular infiltration of the trabeculum or by the formation of a neovascular membrane that covers an OA (this stage can be reversed by appropriate treatment).

Nevertheless, the 2008 EGSc includes the first two manifestations only in primary OAG [POAG] and the reversible stage of neovascular G in ACG.

Thirdly, in addition, some statements in the chapter on secondary OAG arouse additional doubts:

It is stated that in secondary OAG, the angle is open for at least $270^{\circ}$. This statement raises the questions: How can we define an angle closed by $85^{\circ}$ as being open? What justifies the choice of this value? Is there enough difference in $\mathrm{AC}$ between $85^{\circ}$ and $95^{\circ}$ to justify the placement of a case in two opposite categories (OA and $\mathrm{AC}$ )?

Furthermore, the following points have been stated: "In several forms of secondary G, pathomechanisms leading to both secondary OAG and ACG are combined;" "the secondary traumatic $\mathrm{G}$ can be caused by both $\mathrm{OA}$ and $\mathrm{AC}$ pathomechanisms;" and besides "secondary OAG, secondary ACG may also develop.” These statements are true. However, placed in the chapter on secondary OAG, they may confuse a young ophthalmologist who must treat the sickness after placing the case in one of the two opposite categories indicated in the 2008 EGSc. After solving this dichotomy, he would expect it to be easier to reach a therapeutic decision; instead, he finds that the same mechanism may cause both OAG and ACG. As the treatment must target the essential pathogenic link and not the angle conformation, he may consider that all his previous efforts to frame the case in one gonioscopic category did not yield practical results. The immediate consequence may be his inability to understand why the gonioscopic criterion was used for such an important crossing (the first crossing), despite the fact that it cannot make enough difference by itself. The long-term consequence is that the lack of understanding may reduce adherence, and the lack of practical finality may explain why terms like "chronic simple G" and "congestive G" - specific to Donders' clinical classification - are still used.

Finally, on page 104, the Terminology and Guidelines for Glaucoma, ${ }^{11}$ identifies primary angle closure (PAC) suspect, characterized by "two or more quadrants of iridotrabecular contact, normal intraocular pressure (IOP), and absence of peripheral goniosynechiae and glaucomatous optic neuropathy." Yet, a few lines above, there is the following formulation: "An international group of experts reached a consensus that two quadrants or more of iridotrabecular contact is an indication for prophylactic treatment." The abovementioned two statements placed in close succession clearly indicate that when the iridotrabecular contact affects fewer than two quadrants, the prophylactic treatment is not necessary. Such a conception is highly dangerous, especially when it is put forward by the most authorized forum of European glaucoma specialists. ${ }^{11,16}$ Is it justified to postpone prophylactic treatment if we find a narrow angle all around but the iridocorneal contact affects fewer than two quadrants? Is it justified to do so even when an aggravating factor, like evolutive cataract, exists? What significance will the prophylactic treatment have if, on the one hand, we are advised to consider the indication of surgery after a sign that may vary considerably with time, while on the other hand, we are aware that patients cannot be examined at such short intervals that the stage of iridotrabecular contact on two quadrants is observed? The mere suggestion that there are stages in primary angle closure glaucoma [PACG] in which the peripheral iridectomy is not indicated may produce severe consequences. Such a suggestion explains the carelessness with which some doctors recommend chronic topical medication without peripheral iridectomy in chronic PACG or in precrisis (extremely narrow angle with an aggravating factor [such as evolutive cataract or history of ACG among patient's consanguineous relatives]), although they know that this treatment cannot prevent closure. This is the cause of too many attacks of PACG in patients who have complied with the recommended medical treatment. The only problem lies in the fact that the treatment was recommended on the basis of erroneous conceptions that were accepted uncritically, which the 2008 EGSc further strengthens, instead of amending. 


\section{The 2008 EGSc uses criticizable secondary criteria}

In a good classification, all criteria must be used in such a way that the potential criticism is minimized. This rule is not followed by the 2008 EGSc, which uses many secondary criteria in a criticizable manner.

At the second crossing, the only secondary criterion used under "2.1 Primary congenital G" is the absence or presence of associated congenital anomalies, despite the following facts: that angle dysgenesis inducing "2.1.1 - Primary congenital G/childhood G" (page 93) is also a congenital anomaly, like those mentioned under "2.1.2 G associated with congenital anomalies (page 94);" that terms like "angle dysgenesis" (page 93) and "goniodysgenesis (page 94)," which have been used to define two different categories, have the same meaning; that most entities listed in the chapter "2.1.2 G associated with congenital anomalies" share the fact that $\mathrm{G}$ is not a constant manifestation, while the associated congenital anomalies are always present and characterize the sickness; and that in several of the entities listed at page 94, the "associated congenital anomalies" do not influence the ocular hypertension mechanism, the one that must be targeted by our therapeutic means.

Thus, the use of the abovementioned secondary criterion in the classification of congenital Gs will neither clarify the ocular hypertension mechanism, nor facilitate therapeutic decision making or prevent therapeutic errors. It would have been much more beneficial to use secondary criteria with ophthalmic significance; thus, angle conformation affects our therapeutic decision, while the "primary-secondary" dichotomy may influence parental counseling concerning future pregnancies for a couple who has produced a child with congenital G. A third criterion with ophthalmic significance is the pathogenic one. Although the 2008 EGSc has not even attempted to use it for congenital G, this criterion has the most important impact on therapy, as the treatment must target all pathogenic links.

On page 98 the initial statement that the secondary OAG classification "is primarily based on pathophysiologic mechanisms" is not true for many of the described forms. Only inflammation, effect of long-term corticotherapy, angle recession, increase in episcleral vein pressure, and trabecular obstruction represent such mechanisms. The other listed forms illustrate the last mechanism, and the differentiation is based on obstruction materials. Nevertheless, the materials represent etiologic agents and not pathophysiologic mechanisms.

On page 106, the third crossing uses clinical manifestations for PACG differentiation (acute closure, intermittent closure, chronic closure, status after acute closure, and risk of closure). These are only clinical stages of $\mathrm{AC}$, which do not influence the treatment because in all the stages, the pretrabecular block, if persisting, must be released. Moreover, the described stages are not specific for PACG: some secondary ACGs may pass through some of these stages too.

On page 109, the manner in which the 2008 EGSc uses the pathogenic criterion for the differentiation of ACG is criticizable because it is not valid for all ACG forms. Only secondary ACGs are divided into forms with pupillary block, with pulling mechanism or with pushing mechanism, while PACG is left undifferentiated. The underlying cause is that the suggested terms are not valid for PACG, which has forms without pupillary block, without pulling mechanism, and without pushing mechanism.

I used more comprehensive terms in my pathogenic classification of Gs: "pupillary G," defined as occurring when the angle is closed by some factor acting in the pupil; "angular G," defined as occurring when the angle is closed by some factor acting in the angle itself; and "posterior push G," defined as occurring when the causative factor acts behind the lens-zonule plane. ${ }^{12}$ Although "pulling" as opposed to "pushing" sounds better regarding terminology, "angular" is more comprehensive, because it may be used not only for secondary ACG forms, but also, for the primary ones. I will develop this idea in my next paper.

\section{The 2008 EGSc uses several criteria for one single crossing}

Logically, a classification is good, clear, and useful when it selects only one criterion as fundamental and constantly uses it until its differentiation capabilities are exhausted. Thereafter, a secondary criterion must differentiate the resulting categories, until its capabilities are in turn, exhausted. This procedure should continue until each clinical form finds its natural place. The main target of this reproach is the fact that the 2008 EGSc uses three completely different but equally weighted criteria not only at the first crossing (genetic, etiologic, and gonioscopic - as discussed previously) but also when it tries to classify POAG: age (when it describes primary juvenile G), IOP (when it describes hypertensive and normotensive $G$ ), and lack of enough symptoms for a positive diagnosis (when it refers to POAG suspect and to ocular hypertension). When the authors agreed to use several criteria for one single crossing, they indirectly admitted that they did not find the essential and defining criterion that can frame all forms gathered at that particular level of classification. This poses a major risk: too many criteria used at the same time for the same crossing may confuse not only 
the young and inexperienced ophthalmologists but also the experienced ones, who may be inclined to abandon the classification that did not help them enough. I will return to this idea at the end of this paper.

\section{The 2008 EGSc is not consistent in using a criterion}

Logically, a classification will convey the sensation of unity and will be clear and easy to use only when the same criterion will be applicable to all the groups already described at the previous level of analysis. The 2008 EGSc does not comply with this rule.

I have already mentioned the fact that the gonioscopic and etiologic criteria are used as the fundamental criteria for acquired $\mathrm{G}$ but are not used for congenital $\mathrm{G}$, although they have clinical significance.

On the other hand, a new criterion (association with congenital anomalies) was introduced in the chapter on primary congenital $\mathrm{G}$, although this criterion has limited significance for congenital $\mathrm{G}$, and a similar criterion was not used to classify acquired Gs.

The 2008 EGSc has rejected the "age" criterion for congenital $\mathrm{G}$, although it is used for acquired $\mathrm{G}$. In the chapter on POAG, the hypertensive juvenile and hypertensive adult forms are described as forms with their own identities, although besides age, there are no other significant clinical differences. It is difficult to understand why age has no significance regarding congenital $\mathrm{G}$, where infantile $\mathrm{G}$ has lost its identity, although besides age, there are other significant clinical differences when compared with the proper primary congenital G. (In infantile G, there is no buphthalmia or Descemet membrane ruptures; the eye does not show progressive myopia; the optic nerve head shows more excavation and less atrophy; and the response to surgical treatment can be more favorable.) In spite of these differences, the infantile $G$ has been included in the group "primary congenital G/childhood G" - the first "camel-ostrich" in the 2008 EGSc ["camel-ostrich" is a Romanian expression defining unmixable items, put together against nature].

Another inconsistency is that the pathogenic criterion is used for some acquired secondary ACGs but is not used for congenital $\mathrm{G}$, although some congenital $\mathrm{G}$ forms might suggest its use: microspherophakia, microcornea, microphthalmia, and nanophthalmia could populate the group of congenital ACGs with pupillary block; neovascular G secondary to angiogenic factor release from the ischemic retina (in retinoblastoma, medulloepithelioma, Coats' disease, familial exudative vitreoretinopathy, old retinal detachment) could exemplify the group of congenital Gs with pulling mechanism; persistent hyperplasic primary vitreous could fit the subgroup of congenital $\mathrm{G}$ with pushing mechanism.

At the third crossing, when the 2008 EGSc tries to classify acquired $\mathrm{G}$, the pathogenic criterion is not used for the differentiation of PACG, although it is largely used for secondary ACG and despite that PACG has three forms with specific pathogenic mechanisms: most forms of PACG show pupillary block mechanism, while one form shows a pushing mechanism (malignant $\mathrm{G}$, which may also manifest itself as a primary form). ${ }^{16}$ Even if it cannot be framed in the "pushing-pulling" scheme, there exists a third form that shows a third pathogenic mechanism (plateau iris $G$ ). Unfortunately, the 2008 EGSc does not use the pathogenic criterion for PACG; as a consequence, in this group, there are crowded forms with different pathogenic mechanisms and sometimes opposite treatments. Thus, the possibility of therapeutic errors discussed in the section "The 2008 EGSc as one step ahead of the previous classifications" still exists because this classification remains gonioscopic when it deals with PACG.

\section{The 2008 EGSc denies the reality in some aspects}

Several examples illustrate this shortcoming.

Associating tissue resistance alteration only with POAG denies the reality, which shows that identical levels of ocular hypertension may exert different effects on the visual field and optic nerve head, not only in POAG, but also, in PACG or secondary $\mathrm{G}$. In reality, the alteration in tissue resistance to pressure aggression is not associated, in any way, to the iridocorneal angle or etiology. Nevertheless, the 2008 EGSc denies this reality and does not even allow the consideration of neuroprotection in PACG or in secondary G. As a consequence, any symposium on neuroprotection specifically mentions "neuroprotection in POAG."

Associating tissue resistance alteration only with low pressure again denies the reality, which shows that similar levels of ocular hypertony may produce similar levels of glaucomatous alteration at various time intervals, or different levels of glaucomatous alteration at similar time intervals.

Associating exogenous $\mathrm{G}$ only with OA does not reflect reality, which shows that extraocular diseases that increase the episcleral vein pressure may occur with any degree of the iridocorneal angle. Nothing prevents such cases from developing ACG. The opposite situation is true, in turn: the presence of chronic ACG does not protect the patient against the majority of exogenous $G$ forms. If an eye with already diagnosed chronic PACG develops exogenous G, can we frame the case in the POAG group only because the 2008 
EGSc has stated that exogenous G may exist only as a form of POAG? The answer is "certainly not," and the explanation is simple: episcleral vein pressure is not influenced by angle configuration.

Placing all stages of neovascular $\mathrm{G}$ in the group of secondary ACG with pulling mechanism, caused by annular synechia, is an error contradicting reality, which shows that the incipient stages may be cured by procedures annulling the vasoformative factor. These procedures cannot influence the already established goniosynechiae; such goniosynechiae can be released only by specific surgical procedures ("goniosynechialysis"17 or "angle repermeation"18). Under these conditions, where can we place the incipient stages of neovascular G? The angle is open in the incipient stages, so these stages should be discussed with other forms of OAG.

Plateau iris $\mathrm{G}$ is a reality that does not fit into any of the three categories suggested by the 2008 EGSc for secondary ACG: in this form, there is no traction, no pushing, and certainly, no pupillary block. Probably because of this sickness, the pathogenic criterion was not used in PACG, and the whole group has remained undifferentiated in the 2008 EGSc. As a result, different forms of sickness having different mechanisms and sometimes even opposite treatments, remain mixed together.

\section{The 2008 EGSc frames a sickness} in more than one category

Sickness is a clinical entity, and the gonioscopic classification is a clinical classification, delimitating clinical categories. We cannot place the same clinical entity in several clinical categories of a clinical classification without making it difficult for anyone inclined to adopt this classification and without reducing the adherence of potential users. We have already discussed that in order to agree with the reality, the 2008 EGSc should have placed neovascular G in two different categories (the incipient stages in $\mathrm{OAG}$ and the advanced stages in ACG).

Unfortunately, this classification shows many other instances where the same sickness is placed in 2 or even 3 categories:

- Sturge-Weber syndrome is classified not only as primary congenital $\mathrm{G}$ associated with congenital anomalies (page 94) but also as secondary acquired OAG (page 102);

- Löwe and Pierre Robin syndromes, persistent hyperplastic primary vitreous, homocystinuria, and goniodysgenesis are classified not only as primary congenital $\mathrm{G}$ associated with congenital anomalies (page 94) but also as secondary ACG with pushing mechanism (page 110);
- Weill-Marchesani syndrome is classified as primary congenital $\mathrm{G}$ associated with congenital anomalies on page 94 but as secondary ACG with pupillary block on page 109;

- Marfan's syndrome and microspherophakia are classified as primary congenital $\mathrm{G}$ associated with congenital anomalies on page 94 and as acquired secondary ACG with papillary block on page 109, while on page 110 , they are classified as acquired secondary ACG with pushing mechanism;

- Aniridia is classified as primary congenital $G$ associated with congenital anomalies on page 94 and as acquired secondary ACG with pulling mechanism on page 109 , while on page 110, it is classified as acquired secondary ACG with pushing mechanism.

\section{The 2008 EGSc does not allow the framing of all forms of sickness}

The question is asked: How can we define, the following forms, using short and clear diagnostic terms formulated according to the $2008 \mathrm{EGSc}$, in such a manner that their framing in the scheme be eased, and the decision making be simplified?

Typical optic nerve head and perimetric alterations with high IOP produced by trabecular obstruction on a narrow angle might be included in ACG, but this term is incorrect, as the angle is not closed and the mechanism is trabecular obstruction; it might also be included in $\mathrm{OAG}$, but this term is incomplete, as it neglects the important information that the angle is occludable in the affected eye, in the opposite eye, or that the condition may occur in consanguineous relatives. The 2008 EGSc recommends the term "OAG and narrow angle;" however, this term is not only confusing, but also, is contradicted by the information in the 2008 EGSc brochure itself, which on page 97 it states that "OA excludes intermittent closure." According to this, reformulation of the term recommended by the 2008 EGSc will produce a second camel-ostrich, ie, $\mathrm{G}$ with an angle that excludes closure but can close sometimes.

Let us say that we accept the camel-ostrich. Then, how can we define a case of "POAG and narrow angle" presented in the hospital in a full AC attack in the left eye? How can the ambulatory doctor, who has followed up the case, formulate the diagnostic term such that the surgeon is informed from the first that the trabeculum is altered under the iris block? How could the surgeon be informed that peripheral iridectomy will not be enough and that the opposite eye is not prone to the risk connected with angle narrowness alone? The 2008 EGSc allows only the term "POAG and AC" - the third camel-ostrich. 
How can we define residual G caused by secondary trabecular meshwork alteration after opening of an angle that has remained closed for a very long time? The term "ACG" is incorrect because the angle is no longer closed. The term "OAG" is incomplete, as the information about an occludable angle in the other eye or in consanguineous relatives is lost.

How can we define three cases with hypertensive POAG? The first case has reached the final stage (temporal island) in 3 years, with an IOP around $24 \mathrm{~mm} \mathrm{Hg}$; the second case has reached the final stage in 12 years, with an IOP around $30 \mathrm{~mm} \mathrm{Hg}$; while the third case shows only Bjerrum scotoma erupted at the periphery after 10 years, with an IOP of around $30 \mathrm{~mm} \mathrm{Hg}$. These three examples are of real cases, taken into evidence in an incipient stage of the sickness and correctly investigated as part of a $\mathrm{PhD}$ thesis, with monthly IOP measurement, tonometric curve every 3 months, and perimetric investigation every 6 months. The patients have refused to undergo surgery but have complied with the prescribed medication.

The case differences explained above are important because the doctor who receives the patient must be informed from the moment he reads the diagnostic term that the first case must be managed at shorter intervals, including perimetric examination each time. The treatment must be more aggressive, aiming to lower levels of IOP. If surgery is necessary, it must be performed closest to the moment when the case shows progression under maximal medical therapy. The patient must be made aware that in his form of sickness, surgery postponement for a few weeks, which is admissible in other forms, may have disastrous effects. In such a case, in order to lower the IOP to around $11-13 \mathrm{~mm} \mathrm{Hg}$, the surgery must be accompanied by the administration of fibrogenesis inhibitors (mitomycin C, 5-fluorouracil) and fibrogenesis modulators $\left(\mathrm{Ologen}{ }^{\mathrm{TM}}, \mathrm{iGen}^{\mathrm{TM}}\right)$. On the other hand, the third case warrants a completely different attitude: if there are associated cardiovascular complications and excessive surgical risk, the operation may be postponed until the cardiovascular complications are managed. If life expectancy is low, surgery may be completely avoided, as this case showed slow progression. Nevertheless, the follow up must rigorously continue because in debilitated patients, the tissue resistance to high IOP may show sudden reduction. Obviously, the 2008 EGSc cannot differentiate among these three cases, considering all as cases of hypertensive POAG.

How can we define two hypothetical cases of PACG in attack? The first case remains blind at 24 hours, with an
IOP of around $50 \mathrm{~mm} \mathrm{Hg}$, while the second case, after 1 week, with an IOP around $50 \mathrm{~mm} \mathrm{Hg}$ maintains or recovers useful vision. How can we suggest that in the first case, AC may have disastrous effects on the opposite eye? How can we suggest that in this case, peripheral iridectomy on the opposite eye should be performed as soon as possible, even before the painful eye is operated (this eye might have already been lost)? An additional question refers to the consanguineous relatives of the first case - who may be showing narrow angle, too. Not all such relatives will develop the attack in a predictable period, but the risk rises with age. How can we suggest to the surgeon that the safe approach is to perform peripheral iridectomy in all the consanguineous relatives of the first case who are showing narrow angle and aggravating factors (eg, incipient cataract), even if the provoking test results remain negative for the moment? This question "holds water," as alteration in tissue resistance to pressure aggression may be genetically transmitted, which is also suggested by the familial incidence of normotensive G. ${ }^{19}$ On the other hand, in many regions of the world, in vain, frequent monitoring is recommended in order to observe the moment when the closure risk becomes significant. The patients usually visit only days or even weeks after they first experienced pain. If the attack occurs in patients with altered tissue resistance in front of pressure aggression, the consequences might be disastrous, as in the first case.

Unfortunately, nowadays there are no means to prospectively measure the level of tissue resistance to pressure aggression. Therefore, we must be content with the retrospective information provided by this first patient that the resistance to pressure aggression might be altered among his consanguineous relatives: in order to avoid disastrous consequences, it is safer to perform peripheral iridectomy in all the consanguineous relatives showing narrow angle and aggravating factors, even if the provoking tests results have remained negative for the moment.

Obviously, the 2008 EGSc cannot differentiate among these cases either, and the reason is that this classification associates alteration in tissue resistance only with POAG, neglecting its influence in PACG or secondary G.

And finally, there is the question: Where can we place the mixed G forms in the 2008 EGSc? Where can we place: secondary phacomorphic ACG, which may complicate the evolution of exogenous $\mathrm{G}$; exogenous $\mathrm{G}$ due to severe alkali burns, occurring in a case of chronic ACG; ACG, which may complicate the evolution of OAG; and hypertensive OAG, which may complicate the evolution of normotensive OAG, and how can we convey 
the information that the sickness is much more severe than suggested by the IOP level? These questions remain unresolved as the 2008 EGSc cannot offer any answer.

\section{The 2008 EGSc does not offer direct therapeutical suggestions}

In the middle of the last century, although $\mathrm{G}$ had one of the richest therapeutic arsenal of the whole ophthalmic pathology (with its several hundred of described surgical procedures), there were few generally accepted therapeutic options. At the same time, the pathogeny of most cases was obscure; hence, the therapeutic choice was simple, even if it was guided by the new gonioscopic classification (which is, in fact, very close to the former, clinical one).

Beginning with the eighth decade, the knowledge level largely increased. Consequently, by the end of the century, the pathogenic mechanism had been understood for almost every form, with a specific treatment for almost every mechanism. Unfortunately, the gonioscopic classification remained "blocked in its project," [a system may be open or "blocked in its project", confined to its limits]. The present paper is full of examples in which new scientific achievements cannot be covered by gonioscopic classification. This classification describes only two main forms of $\mathrm{G}$, while the doctor had to choose from a much larger palette of options. Therapeutic decision making was not facilitated in any way, and errors leading to disasters remain possible: pilocarpine drops, iridectomy, or trabeculectomy - in malignant G; trabeculectomy or iridectomy - in secondary-to-tumors $\mathrm{G}$ or in neovascular $\mathrm{G}$ (before annulling the vascular endothelial growth factor); iridectomy - in OAG or plateau iris G; and long-term topical treatment without iridectomy - in chronic $\mathrm{ACG}$ or in precrisis (AC risk with aggravating factors). If the first errors are not frequent, the last one is unexpectedly widespread, indicating that the users will avoid a tool if they find its performance unrewarding. As the gonioscopic classification did not considerably facilitate therapeutic decision making, some doctors have reoriented themselves toward the clinical classifications, using terms like "chronic simple" and "congestive" and prescribing long-term medical treatment without peripheral iridectomy in chronic PACG or in precrisis, only because the eye is quiet.

The importance of this tendency is shown by the fact that there are university staff members who think, write, and act according to Donders' classification. In 2009, I diagnosed a case as "POAG and narrow angle" according to the 2008 EGSc (quiet eyes, very narrow angle all around but without iridotrabecular contact; IOP in the right eye $=28 \mathrm{mmHg}$ and in the left eye $=30 \mathrm{mmHg}$; glaucomatous excavation with a cup/disc ratio of 0.5 for both eyes and asymmetrically narrowed rim; and incipient cataract). I immediately recommended topical triple medication ( $\beta$ blocker plus carbonic anhydrase inhibitor plus prostaglandin derivative) and peripheral iridectomy. Although after 1 week, the IOP had decreased to 16 and $17 \mathrm{~mm} \mathrm{Hg}$ for the right and left eye, respectively, I continued to insist on the necessity of peripheral iridectomy, the only procedure that could prevent $\mathrm{AC}$ risk. The patient sought a second opinion in a university clinic and returned with a note in which the sickness was defined as "chronic simple G" and with the indication to continue the triple medication. After 5 months of compliance with the treatment, the patient was admitted to our hospital with acute ACG in the right eye. I am convinced that the doctor knew that the medication could not prevent AC. Nevertheless, he prescribed it because he had already rejected the gonioscopic classification, the eyes were quiet (as in any case of chronic simple G), and the elective treatment for chronic simple $\mathrm{G}$ - which responds to local medication - is continuation of medication. Thus, influenced by some university staff members, the young ophthalmologist may leave the clinic with wrong thinking habits. Unfortunately, the young ophthalmologist grows old with the same way of thinking, while the 2008 EGSc - instead of bringing clarity in medical thinking - exposes to the same therapeutic errors as those made likely by all the previous classifications.

\section{Miscellanea that might confuse young ophthalmologists}

The absence of a definition at the beginning of any new chapter is particularly evident in the chapter on congenital G. In the literature, most authors sustain that congenital $\mathrm{G}$ is the form that occurs before the age of 2 years, manifested by buphthalmia and determined by an increase in the IOP on an elastic sclera. If we accept this definition, then many forms listed under "2.1.2 G associated with congenital anomalies," in which high IOP appears after the age of 2 years, are not the forms of congenital $G$ because buphthalmia is absent and alterations in the optic nerve head are of the type appearing in adults. The authors of the 2008 EGSc seem to favor the opposite theory, which defines congenital $\mathrm{G}$ as the form of sickness caused by congenital anomalies existing at birth, even if the IOP rises later, on a sclera that has become rigid. This definition is not clearly stated anywhere in the brochure; it is only suggested by the fact that infantile $\mathrm{G}$ is mentioned in a single line at the end of the chapter "2.1.1 Primary congenital G/childhood G." 
This means that buphthalmia and IOP that rise in the first 2 years after birth are no longer the defining characteristics of congenital G. As a consequence, most forms listed under "2.1.2 G associated with congenital anomalies" are true congenital Gs. Under these conditions, it is difficult to understand why these forms are listed once or several times in the chapter on acquired G. I do not think that this uncertainty helps the young ophthalmologist.

The absence of a "clear minded" character from the editorial board is another shortcoming that can be discussed here. Many earlier mentioned inadvertencies could be listed in this subchapter and will not be repeated. Nevertheless, there are some inadvertencies that can be discussed only here.

In the chapter "2.1.1 Primary congenital G," the 2008 EGSc includes two subgroups: "2.1.1 Primary congenital $\mathrm{G} /$ childhood G" and "2.1.2 G associated with congenital anomalies." In this numbering system, all the subgroups that belong to the same group should bear a name derived from the main group name. Thus, the name of the second subgroup should have been "2.1.2 Primary congenital G associated with congenital anomalies." Occurring without the prefix "Primary congenital," the name in the brochure may suggest to the young ophthalmologist that $\mathrm{G}$ in these clinical manifestations is no longer primary or congenital, as only the associated anomalies are indicated to be congenital.

The 2008 EGSc describes four pathogenic mechanisms of angle closure in the chapter on PACG (pages 103-108): pupillary block, plateau iris, lens block, and aqueous misdirection. In this regard, it is difficult to understand why the lens block has been described as a form with its own identity when it is caused by pupillary block. Similar to any primary pupillary block, primary block at the level of the lens is caused by the pressure gradient between the anterior and posterior chambers, which is produced by a very close contact between the lens and the iris-pupillary region. Further, similar to any primary pupillary block, primary lens block occurs as a consequence of an inborn disproportion between the lens and the rest of the anterior segment constituents. It is also difficult to understand why the block at the level of the lens is described in the chapter on PACG, when it is said to be frequently caused by pathologic, traumatic, or iatrogenic alterations in lens thickness or position. When we analyze the section on "Obstruction at the level of the lens" (page 105) we find that four of the seven lines refer to such alterations, or that $\mathrm{G}$ caused by such alterations is secondary G, and not primary G. Under these conditions, if the last four lines discuss the secondary phakogenic pupillary block, it is difficult to understand why they have been included in the chapter on primary ACG. If only the first three lines might be included in the chapter on PACG, but describe the same pupillary block mechanism, why has the lens block been indicated as a separate mechanism with its own identity?

In the same chapter, the Terminology and Guidelines for Glaucoma ${ }^{11}$ describes the primary AC mechanisms but does not use them in any way for differentiating primary ACG. On the contrary, it uses only some of these mechanisms for differentiating the secondary ACG forms. This contradiction raises several other questions: If the mechanisms said to differentiate the PACG group do not exert any effects in PACG, why have they been included in the chapter on PACG? If they have been used only for differentiating secondary ACG, why have they not been discussed in the chapter on secondary ACG? If these mechanisms are valid for both forms, why have they not been described in the introductory lines of the chapter on ACG, before referring to the primary and secondary forms?

I doubt if using what has not been described and not using what has been described will help the young ophthalmologist, the more so that four mechanisms were described in one chapter, but only three were used in another chapter.

Further, I am forced to explain why I have repeatedly remarked on the way of thinking of young ophthalmologists: the main addressees of my pathogenic classification are the beginners in ophthalmology. The experienced doctors might have established their own way of thinking and can manage the deficiencies of a classification based on iridocorneal angle aperture. To the contrary, the young ophthalmologist needs a system that must be at the same time logical (based on a coherent set of criteria) and useful from the practical point of view (containing elements intended to facilitate therapeutic decision making). If a classification is not logical, the young ophthalmologist will face difficulties in understanding, receiving, and using it. If the classification does not offer direct therapeutic suggestions, that classification might be rejected, not only by young ophthalmologists but also by university staff members, as proved by the case described earlier, of my patient with POAG and narrow angle. Moreover, if a classification stimulates therapeutic errors, it does not deserve to be used.

A good classification must prevent therapeutic errors, persuading the doctor to analyze the case up to the end before selecting its diagnostic category. This additional diagnostic effort will be rewarded at the stage of therapeutic decision making: after the pathogenic mechanism is understood, the diagnostic category selected, and the case placed in its appropriate group, a good classification will directly suggest the correct treatment, reducing further effort and preventing the risk of therapeutic errors. 
The torrent of questions presented in the previous page was caused by the fact that the authors did not succeed in finding a scheme valid for both primary and secondary forms of ACG. The sickness that does not fit in the scheme defined by pupillary block-pushing-pulling mechanisms is plateau iris G. As I have previously affirmed, probably because of this form, the whole group of PACG has been left undifferentiated. Instead of leaving a whole group undifferentiated because one entity does not fit the scheme built on a certain criterion, it is preferable to use a new criterion and build a new scheme that can completely adhere to the reality. This scheme will be presented in my next paper.

\section{Conclusion}

The 2008 EGSc represents an important step forward because it brings clarity to the group of secondary ACGs, in which forms having different pathogenic mechanisms and sometimes even opposite treatments were previously crowded together.

The 2008 EGSc has most of the other drawbacks of the previous classifications: it uses criticizable criteria in a criticizable manner; it uses several criteria for a single crossing; it is not consistent in its use of a criterion; it uses, from the first crossing, criteria that cannot cover all cases; it describes categories which do not cover all forms; it places the same clinical entity in several clinical categories; it includes stages of illness in a category to which they do not belong. The most serious drawback is that it does not offer direct therapeutic suggestions.

The 2008 EGSc is some steps behind the previous classifications regarding congenital $\mathrm{G}$, for which some useful criteria have not been used, being replaced by criteria lacking ophthalmic significance.

All the above mentioned points represent arguments in favor of continuation of efforts until a new classification is found that is clear, all inclusive, and useful, offering direct therapeutic suggestions.

\section{Disclosure}

The author reports no conflict of interest in this work.

Clinical Ophthalmology

\section{Publish your work in this journal}

Clinical Ophthalmology is an international, peer-reviewed journal covering all subspecialties within ophthalmology. Key topics include: Optometry; Visual science; Pharmacology and drug therapy in eye diseases; Basic Sciences; Primary and Secondary eye care; Patient Safety and Quality of Care Improvements. This journal is indexed on Submit your manuscript here: http://www.dovepress.com/clinical-ophthalmology-journal

\section{References}

1. Donders FC. see Hoffmanns: vGraeffes Arch Ophthal. 1862; $8(2): 124$.

2. Duke-Elder WS Sir, editor. Glaucoma: a Symposium Organized by the Council for International Organizations of Medical Sciences. Springfield: Thomas; 1955.

3. Ourgaud AG, Étienne R. L'Exploration Fonctionelle de l'Oeil Glaucomateux: Rapport de la SFO [The functional investigation of glaucoma eye. Report of the French Society of Ophthalmology]. Paris: Masson; 1961:2. French.

4. Krasnov MM. [Pathogenic forms of glaucoma and principles governing their treatment]. Vestn Oftalmol. 1965;78(5):29-35. Russian.

5. Saraux H, Biais B. Precis d'Ophthalmologie [Precis of Ophthalmology]. Paris: Masson; 1969. French.

6. Etienne R. Les Glaucomes [The Glaucomas]. Marseille: Diffusion Générale de Librairie; 1969. French.

7. Duke-Elder WS Sir. System of Ophthalmology. London: Kimpton; $1969 ; 11$.

8. Bonamour G. Ophthalmologie Clinique [Clinical Ophthalmology]. Paris: Doin; 1969. French.

9. Greve E. Glaucoma Abstracts. Amsterdam: Kugler; 1985-1998.

10. European Glaucoma Society. Terminology and Guidelines for Glaucoma. 2nd ed. Savona: Dogma; 2003.

11. European Glaucoma Society. Terminology and Guidelines for Glaucoma. 3rd ed. Savona: Dogma; 2008.

12. Bordeianu CD. The pathogenic classification of glaucomas. Oftalmologia (Bucureşti). 1992;36(4):331-342.

13. Bordeianu $\mathrm{CD}$. The gonioscopic classification $(\mathrm{GC})$ versus a pathogenic one: past and future in glaucoma classification. XII Congress of the European Society of Ophthalmology, Stockholm, Sweden. 1999:91.

14. Rand Allingham R, Damji KF, Freeman S, Moroi SE, editors. Shields's Textbook of Glaucoma. 5th ed. Philadelphia: Lippincott, Williams and Wilkins, 2005.

15. Vajaranant TS, Al Shahwan S, Bejjani BA, et al. A comprehensive update on congenital glaucoma. Curr Pediatr Rev. 2008;4:19-30.

16. Weinreb RN, Friedman DS, editors. Angle Closure and Angle Closure Glaucoma: Reports and Consensus Statements at the 3rd Global AIGS Consensus Meeting on Angle Closure Glaucoma. The Hague: Kugler Publications; 2006.

17. Campbell DG, Vela A. Modern goniosynechialysis for the treatment of synechial angle-closure glaucoma. Ophthalmology. 1984;91(9): 1052-1060.

18. Bordeianu CD. Angle Repermeation - Surgery of Choice in Neglected Pupillary Glaucomas: In SOE 2003 - Societas Ophthalmologica Europaea, XIV Congress of the European Society of Ophthalmology, Madrid, Spain, June 7-12, 2003. Bologna: Monduzzi Editore; 2003:159-164.

19. Hitchings R. Normal tension glaucoma. In: Yanoff M, Duker JS, editors. Ophthalmology. 2nd ed. St Louis: Mosby; 2004:1488-1489.

\section{Dovepress}

PubMed Central and CAS, and is the official journal of The Society of Clinical Ophthalmology (SCO). The manuscript management system is completely online and includes a very quick and fair peer-review system, which is all easy to use. Visit http://www.dovepress.com/ testimonials.php to read real quotes from published authors. 